\title{
Crystal Morphologies of Organolead Trihalide in Mesoscopic/Planar Perovskite Solar Cells
}

Yuanyuan Zhou, ${ }^{* \dagger}$ Alexander L. Vasiliev, ${ }^{\dagger}, \|$ Wenwen $\mathrm{Wu},{ }^{\dagger}$ Mengjin Yang, ${ }^{\ddagger}$ Shuping Pang, ${ }^{\S}$ Kai Zhu, ${ }^{\ddagger}$ and Nitin P. Padture* ${ }^{\dagger}$

${ }^{\dagger}$ School of Engineering, Brown University, 184 Hope Street, Providence, Rhode Island 02912, United States

*Chemical and Materials Science Center, National Renewable Energy Laboratory, 15013 Denver West Parkway, Golden, Colorado 80401, United States

${ }^{\S}$ Qingdao Institute of Bioenergy and Bioprocess Technology, Chinese Academy of Sciences, 189 Songling Road, Qingdao 266101, P.R. China

\section{Supporting Information}

ABSTRACT: The crystal morphology of organolead trihalide perovskite (OTP) light absorbers can have profound influence on the perovskite solar cells (PSCs) performance. Here we have used a combination of conventional transmission electron microscopy (TEM) and high-resolution TEM (HRTEM), in cross-section and plan-view, to characterize the morphologies of a solution-processed OTP $\left(\mathrm{CH}_{3} \mathrm{NH}_{3} \mathrm{PbI}_{3}\right.$ or $\left.\mathrm{MAPbI}_{3}\right)$ within mesoporous $\mathrm{TiO}_{2}$ scaffolds and within capping and planar layers. Studies of TEM specimens prepared with and without the use of focused ion beam (FIB) show that FIBing is a viable method for preparing TEM specimens. HRTEM studies, in conjunction with quantitative X-ray diffraction, show that $\mathrm{MAPbI}_{3}$ perovskite within mesoporous $\mathrm{TiO}_{2}$ scaffold has equiaxed grains of size $10-20 \mathrm{~nm}$ and relatively low crystallinity. In contrast, the grain size of

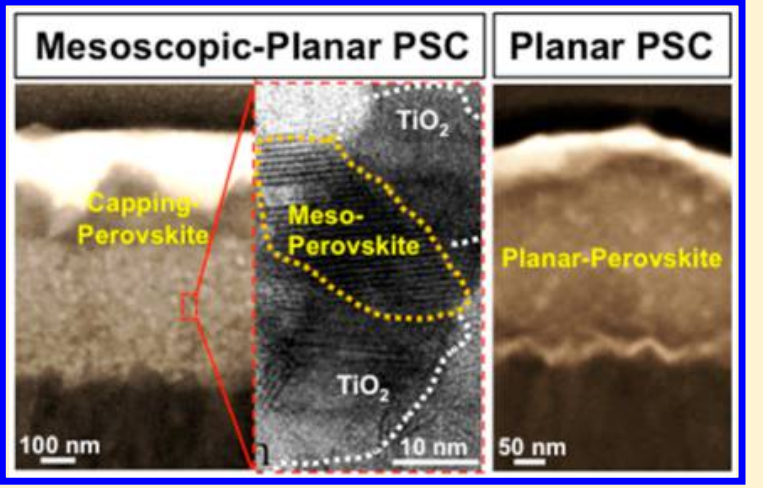
$\mathrm{MAPbI}_{3}$ perovskite in the capping and the planar layers can be larger than $100 \mathrm{~nm}$ in our PSCs, and the grains can be elongated and textured, with relatively high crystallinity. The observed differences in the performance of planar and mesoscopic-planar hybrid PSCs can be attributed in part to the striking differences in their perovskite-grain morphologies.

S olar cells based on solution-processed organometal trihalide perovskite (OTP) have emerged as a new "player" in the photovoltaics (PVs) field over the past few years. ${ }^{1-4}$ Since Miyasaka and coworkers ${ }^{5}$ first reported the use of methylammonium lead triiodine $\left(\mathrm{CH}_{3} \mathrm{NH}_{3} \mathrm{PbI}_{3}\right.$ or $\left.\mathrm{MAPbI}_{3}\right)$ perovskite as light harvester, the power conversion efficiency (PCE) of perovskite solar cells (PSCs) has topped 20\% within a short period of time. ${ }^{6}$ On the basis of the architecture of the photoactive OTP layer, the PSC embodiments are broadly classified as ${ }^{1-4,7}$ (i) mesoscopic, (ii) planar, and (iii) mesoscopic-planar hybrid. In the mesoscopic PSC the photoactive layer consists of a thick (typically 300 to $1000 \mathrm{~nm}$ ) mesoporous oxide $\left(\mathrm{TiO}_{2}, \mathrm{Al}_{2} \mathrm{O}_{3}\right)$ scaffold fully infiltrated by the OTP. The scaffold helps anchor the OTP, and in the case of $\mathrm{TiO}_{2}$ it provides electron-conduction pathways. ${ }^{8}$ The planar PSC is based on a thin film of OTP without the mesoporous oxide scaffold, and it has gained popularity due to the enhanced light absorption and simpler architecture; however, planar PSCs are generally prone to photocurrent $(J)$-voltage $(V)$ hysteresis issues. ${ }^{9}$ The third emerging type of PSCs with mesoscopicplanar hybrid structure aspires to combine the two structures and their desirable attributes into one. These PSCs typically use a mesoporous oxide scaffold that is fully infiltrated by the OTP and then topped with a planar OTP "capping" layer, which enhances light absorption, ${ }^{10,11}$ resulting in PSCs with hysteresis-free PCE of $>20 \%{ }^{12}$

There have been numerous studies on controlling the overall morphology/coverage (see, e.g., ref 13) and crystallinity (see e.g. ref 14) of OTP thin films in the different PSC embodiments and their effects on OTP properties and performance of the PSCs; however, there is paucity of detailed characterization studies of the OTP crystals (grains) themselves within those PSC embodiments. Because the OTP within the oxide-scaffold mesopores crystallizes under a 3-D constraint, which is absent for planar OTP or capping-layer OTP, the size and the morphology of the OTP crystals in those locations are different and thus have different properties. Scanning electron microscopy (SEM) is commonly used to study OTP films, but the SEM has significant limitations in terms of resolution and the ability to identify crystal phases. In this context, Petrozza and coworkers ${ }^{15}$ have characterized $\mathrm{MAPbI}_{3}$ and $\mathrm{MAPbI}_{3-x} \mathrm{Cl}_{x}$

Received: May 12, 2015

Accepted: June 1, 2015

Published: June 4, 2015 
in the form of planar films and inside mesoporous $\mathrm{TiO}_{2}$ using Raman spectroscopy and X-ray diffraction (XRD). They highlight the differences in the way the OTP crystallizes in the two forms and how it affects the onset of optical absorption in OTPs and other properties such as texture. Most recently, Mora-Sero and coworkers ${ }^{16}$ have studied $\mathrm{MAPbI}_{3-x} \mathrm{Cl}_{x}$ in planar films, within mesoporous oxide scaffolds and in mesoscopic-planar hybrid PSCs, using quantitative XRD. They find that the grain size of the OTP within the mesoporous oxides is 25 to $26 \mathrm{~nm}$, consistent with the size of the mesopores, and grain size in both the planar film and the capping layer is $134-150 \mathrm{~nm},{ }^{16}$ although that grain size is beyond the validity of quantitative XRD methods and no direct visual evidence is provided. Using photoluminescence (PL) spectroscopy, they observe dramatic differences in the electron diffusion lengths $\left(L_{\mathrm{D}}\right)$, with $L_{\mathrm{D}}$ values for planar, mesoscopic, and mesoscopic-planar hybrid PSCs of 400, 40, and $260 \mathrm{~nm}$, respectively. ${ }^{16}$ They conclude that the OTP in the mesoporous oxide controls the contact properties, while the OTP capping layer controls the photovoltaic performance. Nanova et al. ${ }^{19}$ have studied mesoscopic-planar $\mathrm{MAPbI}_{3-x} \mathrm{Cl}_{x}$ films using transmission electron microscopy (TEM), in conjunction with electron energy loss spectroscopy (EELS), to overcome some of the limitations of SEM, Raman spectroscopy, and XRD. That study used energy-filtered EELS elemental mapping to deduce the OTP distribution within the $\mathrm{TiO}_{2}$ mesopores and the capping layer, ${ }^{17}$ although there is no mention of OTP grain size and the perovskite phase is not identified as such. Nevertheless, these papers highlight the importance of studying the morphology of OTP grains within the different locations (planar, mesoscopic, capping layer), which can have a dramatic effect on the OTP properties. To that end, we have used a combination of TEM and quantitative XRD techniques, that complement each other, to study the site-specific morphology of $\mathrm{MAPbI}_{3}$ perovskite in mesoscopic, planar, and capping-layer locations.

Figure 1A,B shows cross-sectional bright-field TEM images of mesoscopic-planar hybrid and planar PSCs specimens, respectively, prepared using focused ion beam (FIB), providing overall views of the PSCs. (See Supporting Information (SI) for all experimental details.) In the mesoscopic-planar hybrid PSC (Figure 1A), both the mesoscopic- $\mathrm{TiO}_{2}-\mathrm{MAPbI}_{3}$ and the $\mathrm{MAPbI}_{3}$ capping layer can be seen clearly, although the capping layer is somewhat rough in this particular PSC. The energy-dispersive spectroscopy (EDS) elemental maps (Figure 1C) show significantly reduced $\mathrm{Ti}$ signal (noise) within the capping layer and strong presence of $\mathrm{Pb}$ and $\mathrm{I}$ in both layers. In the case of the planar PSC (Figure 1B), the $\sim 300 \mathrm{~nm}$ planar $\mathrm{MAPbI}_{3}$ layer, in addition to the fluorinated tin oxide (FTO) substrate, the compact- $\mathrm{TiO}_{2}$ layer, the hole-transporting material (HTM) layer, and the metal contact, can be delineated clearly. This is confirmed by elemental ( $\mathrm{Ti}, \mathrm{Pb}, \mathrm{I})$ EDS mapping shown in Figure $1 \mathrm{D}$, where the $\mathrm{Pb}$ and I signals are observed only within the planar layer. Note the dense nature of the planar $\mathrm{MAPbI}_{3}$ layer in Figure 1A and the absence of HTM penetration into that layer, which is attributed to the efficacy of the sequential spin-coating/annealing (SSCA) method ${ }^{18}$ used to prepare these $\mathrm{MAPbI}_{3}$ films (see SI).

To understand the detailed morphology of the $\mathrm{MAPbI}_{3}$ perovskite, we performed HRTEM studies on the TEM specimens from Figure 1A,B. While great care was exercised in preparing these cross-section TEM specimens using FIB (nanomachining using a Ga-ion beam), including the use of

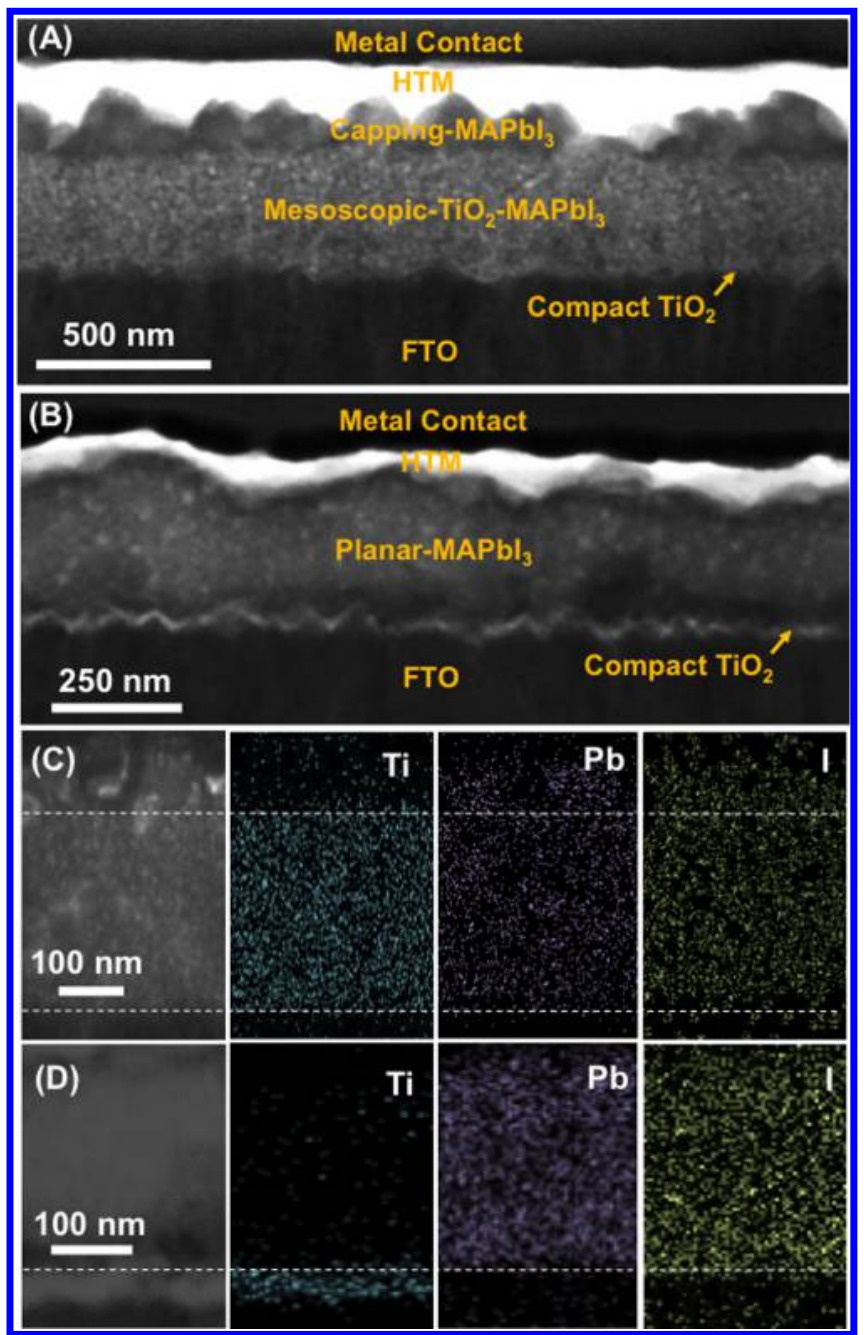

Figure 1. Bright-field TEM images of cross sections of whole PSCs: (A) mesoscopic-planar hybrid and (B) planar. The different layers are identified on the images. STEM images and corresponding EDS elemental maps ( $\mathrm{Ti}, \mathrm{Pb}, \mathrm{I})$ of cross sections of whole PSCs: (C) mesoscopic-planar hybrid and (D) planar. Note the different magnifications. FIBed TEM specimens.

very low beam current $(\sim 10 \mathrm{pA})$ during the final polishing steps (see SI), some damage to the soft $\mathrm{MAPbI}_{3}$ organicinorganic perovskite materials may occur during the FIBing process. To address this issue, we prepared additional TEM specimens without the use of FIB by depositing planar and mesoscopic-planar hybrid $\mathrm{MAPbI}_{3}$ films directly onto TEM grids, and they were observed in plan-view. HRTEM results from FIBed (cross-section) and non-FIBed (plan-view) specimens are presented in Figures 2 and 3, respectively. It is noted that $\mathrm{MAPbI}_{3}$ perovskites are also prone to electron-beaminduced damage and decomposition, and hence it is important to use low beam currents and short exposures times during TEM observation. Thus, unlike in the case of hard inorganic materials, this limits the number of techniques (selected area electron diffraction (SAED), EELS, EDS, etc.) within the TEM that can be applied for comprehensive studies of the same region of the TEM specimens of these soft organic-inorganic materials.

Figure $2 \mathrm{~A}$ is cross-sectional HRTEM image of the $\mathrm{MAPbI}_{3}$ capping layer in the mesoscopic-planar hybrid specimen (FIBed), showing large $\mathrm{MAPbI}_{3}$ perovskite grains. Abrupt 


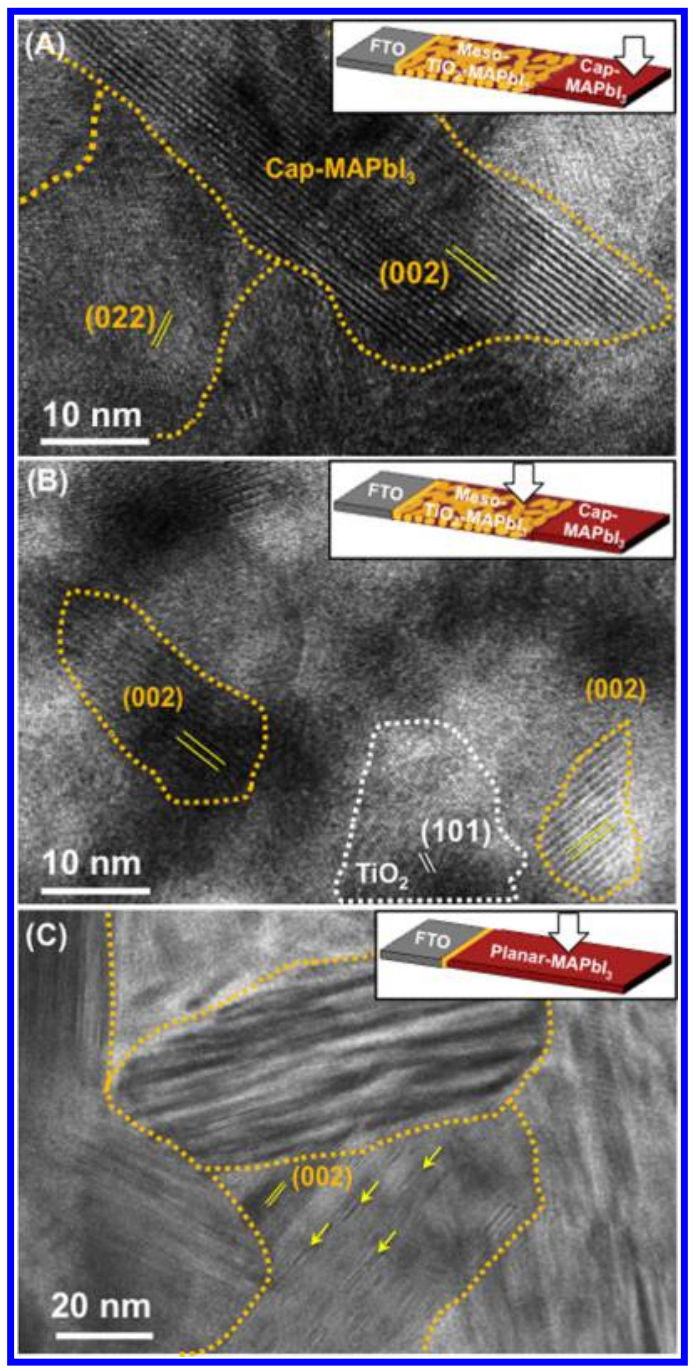

Figure 2. Cross-sectional HRTEM micrographs of different regions in PSCs, as illustrated in the insets: (A) $\mathrm{MAPbI}_{3}$-capping layer in mesoscopic-planar hybrid, (B) mesoscopic- $\mathrm{TiO}_{2}-\mathrm{MAPbI}_{3}$ layer in mesoscopic-planar hybrid, and (C) $\mathrm{MAPbI}_{3}$ in planar. $\mathrm{MAPbI}_{3}$ and $\mathrm{TiO}_{2}$ grains are outlined in yellow and white dashed lines, respectively. Nominal lattice fringe spacings are marked. FIBed TEM specimens.

change in the orientation or termination of lattice fringes is used to estimate the grain boundary locations (dashed lines in Figures 2 and 3). The nominal lattice-fringe spacings are consistent with (002) and (022) planes in $\mathrm{MAPbI}_{3}$ perovskite, as denoted in Figure 2A. (Note that the interplanar-spacings differences between (002) and (110) planes and between (220) and (004) planes are extremely small, and they cannot be resolved in HRTEM.) In contrast, the $\mathrm{MAPbI}_{3}$ perovskite grains within mesoporous $\mathrm{TiO}_{2}$ are much smaller $(10-20 \mathrm{~nm})$, as seen in the cross-sectional HRTEM image in Figure 2B. Once again, the nominal lattice fringe spacing is consistent with (002) planes in $\mathrm{MAPbI}_{3}$ perovskite. $\mathrm{TiO}_{2}$ (anatase) nanograins are also observed in Figure 2B, with (101) lattice spacing resolved. Figure $2 \mathrm{C}$ is a cross-sectional HRTEM image from planar $\mathrm{MAPbI}_{3}$ film (FIBed specimen), showing large defective $\mathrm{MAPbI}_{3}$ grains. Nominal lattice fringes (002) and defects (arrows) could be resolved in Figure 2C. Extensive "streaking" of spots in SAED patterns from similar $\mathrm{MAPbI}_{3}$ TEM specimens (non-FIBed planar) indicates that these defects are

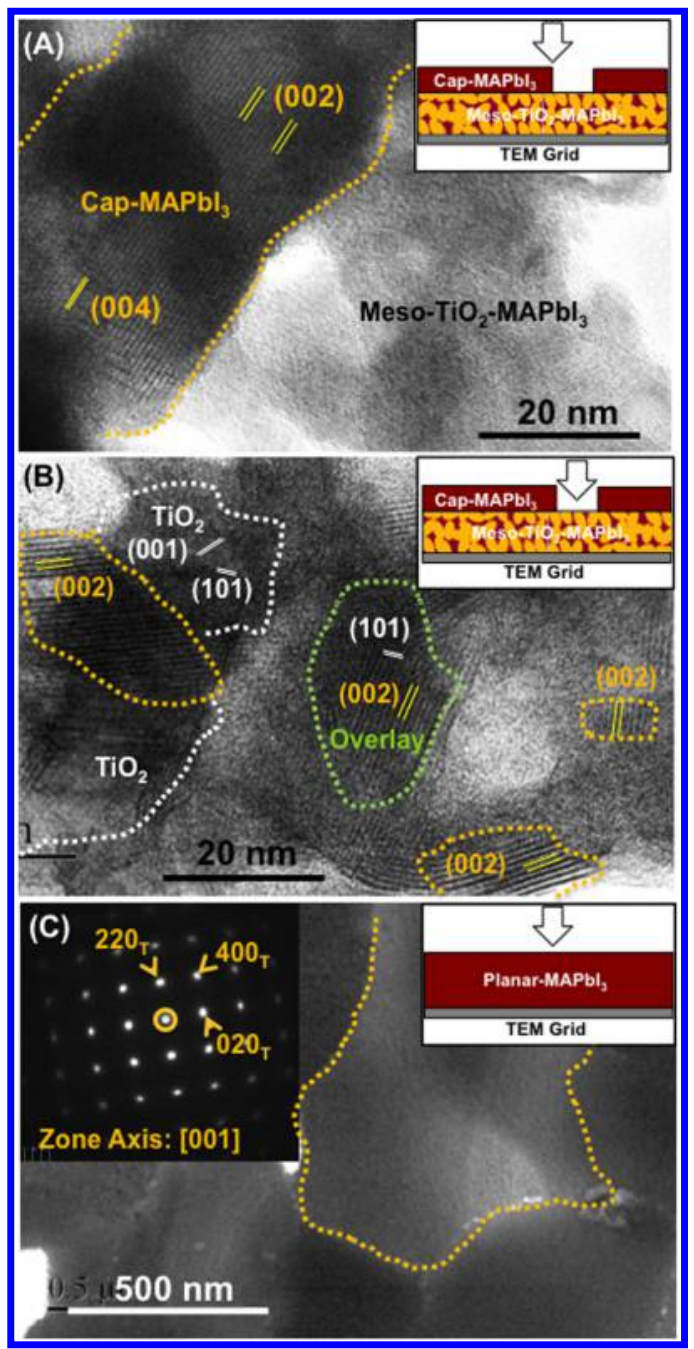

Figure 3. Plan-view HRTEM micrographs of different regions in films deposited on TEM grids, as illustrated in the insets: (A) both $\mathrm{MAPbI}_{3}$ capping and mesoscopic- $\mathrm{TiO}_{2}-\mathrm{MAPbI}_{3}$ layers in mesoscopic-planar hybrid, (B) mesoscopic- $\mathrm{TiO}_{2}-\mathrm{MAPbI}_{3}$ layer in mesoscopic-planar hybrid, and (C) $\mathrm{MAPbI}_{3}$ in planar. $\mathrm{MAPbI}_{3}$ and $\mathrm{TiO}_{2}$ grains are outlined in yellow and white dashed lines, respectively. Green dashed lines denote overlay of $\mathrm{MAPbI}_{3}$ and $\mathrm{TiO}_{2}$ grains. Nominal lattice fringe spacings are marked. Inset in panel $\mathrm{C}$ is an indexed SAEDP ([001] zone axis, transmitted beam denoted by the circle) from $\mathrm{MAPbI}_{3}$ grain confirming the tetragonal perovskite phase. TEM specimens not FIBed.

planar faults (see SI Figure S1), but the exact nature of these faults is not known at this time.

Figure $3 \mathrm{~A}$ is a plan-view HRTEM image of a region containing both the capping layer and the mesoscopic $\mathrm{MAPbI}_{3}$ perovskite (non-FIBed). Relatively large $\mathrm{MAPbI}_{3}$ perovskite grains are observed in the capping layer. The mesoscopic region is not well-resolved in Figure 3A, but in the image (Figure 3B) from the mesoscopic region small $\mathrm{MAPbI}_{3}$ perovskite grains are observed, with resolved (002) lattice spacings. Anatase $\mathrm{TiO}_{2}$ nanograins are also discernible in Figure 3B. The HRTEM image of the planar $\mathrm{MAPbI}_{3}$ film (non-FIBed) is presented in Figure 3C, showing large $\mathrm{MAPbI}_{3}$ grains. (These grains are larger than those in the planar PSCs (FIBed), which is associated with the differences in the wetting behavior of the precursor solution on the holey-carbon TEM grid and the compact $\mathrm{TiO}_{2}$ surfaces.) Indexed SAEDP from that grain is 
included in Figure 3C inset, confirming the $\mathrm{MAPbI}_{3}$ perovskite phase.

Qualitatively, the HRTEM results in both Figures 2 and 3 show larger grains in the capping layer and the planar $\mathrm{MAPbI}_{3}$ perovskite films, whereas the $\mathrm{MAPbI}_{3}$ perovskite grains within the mesoporous $\mathrm{TiO}_{2}$ are much smaller. These data could not be quantified due to the complex nature of the microstructures. Unlike hard inorganic materials, where crystal (grain) interiors are relatively defect-free, $\mathrm{MAPbI}_{3}$ perovskite grains appear to be replete with lattice defects, making it difficult to demarcate grain boundaries. This appears to be a typical feature of these soft organic-inorganic hybrid materials. ${ }^{18-20}$ Because the defects are observed in both FIBed and non-FIBed TEM specimens, they are not an artifact of the FIBing process. The absence of other discernible differences between the FIBed and the non-FIBed TEM specimens indicates that carefully performed FIBing does not damage the $\mathrm{MAPbI}_{3}$ perovskite significantly, making FIBing a viable TEM-specimen-preparation method for the TEM characterization of PSCs.

Quantitative XRD analysis is used to corroborate the HRTEM results. XRD pattern ( $2 \theta$ range 23.75 to $25.75^{\circ}$ ) from the mesoscopic-planar hybrid film is shown in Figure 4A, containing the 022 reflection from $\mathrm{MAPbI}_{3}$ perovskite (tetragonal, space group $14 / \mathrm{mcm}$ ) and the 101 reflection from



Figure 4. XRD patterns from (A) mesoscopic-planar hybrid, (B) mesoscopic-planar hybrid with the capping layer removed, and (C) planar. The deconvoluted curves are denoted by different colors. Vertical dashed lines denote peak positions for mesoscopic $\mathrm{MAPbI}_{3}$, planar $\mathrm{MAPbI}_{3}$, and $\mathrm{TiO}_{2}$. anatase $\mathrm{TiO}_{2}$. Contributions from capping-MAPbI ${ }_{3}$, mesoscopic- $\mathrm{MAPbI}_{3}, \mathrm{TiO}_{2}$, and the background are deconvoluted in Figure 4A. The signal is dominated by the relatively narrow 022 peak from the capping- $\mathrm{MAPbI}_{3}$ perovskite, followed by a broad 022 peak from the mesoscopic- $\mathrm{MAPbI}_{3}$ perovskite shifted to lower $2 \theta$. To obtain XRD patterns from only the $\mathrm{TiO}_{2}-\mathrm{MAPbI}_{3}$ mesoscopic layer within the mesoscopic-planar hybrid films, we removed the capping layer by polishing it away gently. The XRD pattern in Figure 4B from that layer shows a similarly broad $022 \mathrm{MAPbI}_{3}$ perovskite peak, together with the broad $101 \mathrm{TiO}_{2}$ peak. Using calibrated Scherer equation (see SI) ${ }^{21}$ the mesoscopic- $\mathrm{MAPbI}_{3}$ grain size is estimated to be $\sim 20 \mathrm{~nm}$, which is consistent with the size of $\mathrm{TiO}_{2}$ mesopores. The size of the $\mathrm{TiO}_{2}$ grains from the broadening of its 101 peak (anatase phase) is also estimated at $\sim 20 \mathrm{~nm}$, which is about the size of the particles in the paste used to prepare the mesoscopic $\mathrm{TiO}_{2}$ scaffold. Thus, the quantitative XRD grain size of mesoscopic$\mathrm{MAPbI}_{3}$ is consistent with that observed in the TEM studies. Figure $4 \mathrm{C}$ is $\mathrm{XRD}$ pattern from planar- $\mathrm{MAPbI}_{3}$, which is similar to that of the capping- $\mathrm{MAPbI}_{3}$ (Figure 4A). The Scherer equation could not be used reliably to estimate the grain size of the $\mathrm{MAPbI}_{3}$ perovskite in the capping and the planar layers as it can be larger than $100 \mathrm{~nm}$ and is beyond the validity of this quantitative XRD method.

The mesoscopic- $\mathrm{MAPbI}_{3}$ and planar- $\mathrm{MAPbI}_{3}$ peaks are marked by dashed lines in Figure 4, showing a shift to lower $2 \theta$ (by $\sim 0.075^{\circ}$ ) for the mesoscopic-MAPbI . Such as shift is also reported by Mora-Sero and coworkers, ${ }^{16}$ but no explanation is provided. A shift to lower $2 \theta$ implies increase in lattice parameters, that is, a volume expansion of the lattice by $\sim 1 \%$, corresponding to peak shift of $\sim 0.075^{\circ}$. This is consistent with isostatic tensile strain produced in crystals that are grown from liquid precursors inside pores. ${ }^{22}$ The genesis of this tensile strain is the constraint imposed on crystal shrinkage during crystallization by the attachment of the crystal to the pore walls. While the tensile strain in mesoscopic-MAPbI remains to be quantified in detail, it could be responsible for the extended absorption in near-infrared region by mesoscopic$\mathrm{MAPbI}_{3}{ }^{15}$ which is typically not observed in planar-MAPbI ${ }_{3}$.

It has been shown that $J-V$ responses of planar and mesoscopic-planar hybrid PSCs are significantly different, ${ }^{23}$ which is confirmed in Figure 5. In the case of planar PSCs, hysteresis between forward $\left(J_{\mathrm{SC}} \rightarrow V_{\mathrm{OC}}\right)$ and reverse $\left(V_{\mathrm{OC}} \rightarrow\right.$ $\left.J_{\mathrm{SC}}\right)$ scans is typical, as is observed in Figure 5: the forward (F) and reverse $(\mathrm{R})$ scan PCEs are measured to be 8.1 and $12.1 \%$,

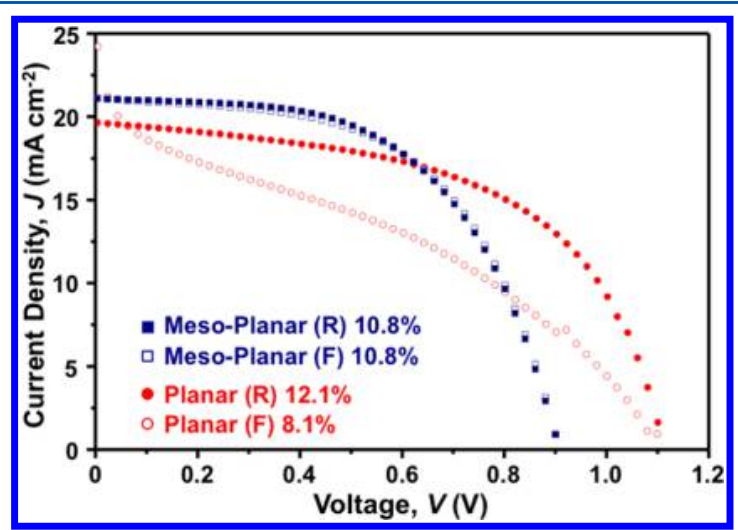

Figure 5. $J-V$ characteristics of typical planar and mesoscopic-planar hybrid PSCs under forward (F) and reverse (R) scans. 
respectively. In contrast, Figure 5 shows that the mesoscopicplanar hybrid PSC is free of hysteresis, with a PCE of $10.8 \%$ regardless of the scan direction. Several hypotheses have been proposed to explain the hysteresis in planar PSCs and its elimination with the addition of the mesoscopic perovskite layer. They include: ${ }^{24-27}$ (i) ferroelectric switching of domains in the perovskite, (ii) ion migration and charge buildup at interfaces, and (iii) crystallographic domain texture in the perovskite. It has been suggested that for $\mathrm{MAPbI}_{3}$ perovskite inside mesoporous $\mathrm{TiO}_{2}$ ferroelectric domain switching is constrained. $^{27}$ Also, the presence of the rough interface between mesoporous $\mathrm{TiO}_{2}$ and $\mathrm{MAPbI}_{3}$ perovskite interface helps screen charge accumulation. ${ }^{24}$ Furthermore, unlike capping- and planar- $\mathrm{MAPbI}_{3}$, mesoscopic- $\mathrm{MAPbI}_{3}$ perovskite grains are less textured. ${ }^{15} \mathrm{~A}$ combination of these attributes is argued to be responsible for the absence of hysteresis when a mesoscopic-MAPbI ${ }_{3}$ layer is included in the PSC. While the overall PCEs of both cells are reasonable, the open-circuit voltage $\left(V_{\mathrm{OC}}\right)$ of planar PSCs is higher $(1.12 \mathrm{~V})$ than that of mesoscopic-planar PSC (0.91 V) (Figure 5), which has been frequently observed by others. ${ }^{1}$ It has been suggested that the high density of the planar perovskite film is more effective in preventing the HTM from making contact with $\mathrm{TiO}_{2} \cdot{ }^{13,18,20}$ In contrast, the capping- $\mathrm{MAPbI}_{3}$ perovskite layer in the mesoscopic-planar hybrid PSC is generally less dense, as seen in Figure 1, and also the surface area of the mesoporous $\mathrm{TiO}_{2}$ layer is very high. These factors enhance the possibility of the formation of parallel $\mathrm{HTM}-\mathrm{TiO}_{2} p-n$ junctions, thereby decreasing $V_{\mathrm{OC}}$ in the mesoscopic-planar PSCs. ${ }^{13,18,20,28}$ This is consistent with high $V_{\mathrm{OC}}$ recorded in our planar PSCs containing high-density $\mathrm{MAPbI}_{3}$ perovskite thin films. ${ }^{18,20}$ This indicates that the $V_{\mathrm{OC}}$ of mesoscopic-planar hybrid PSCs can be enhanced by making the OTP capping layer more dense.

In summary, we have used a combination of conventional TEM and HRTEM, in cross-section and plan-view, to characterize solution-processed $\mathrm{MAPbI}_{3}$ perovskite inside mesoporous $\mathrm{TiO}_{2}$ scaffolds and outside (capping and planar layers). We show that FIBing is a viable method for preparing TEM specimens without significant damage to the $\mathrm{MAPbI}_{3}$ perovskite. The TEM and HRTEM results are supported by quantitative XRD characterization. We show that $\mathrm{MAPbI}_{3}$ perovskite within mesoporous $\mathrm{TiO}_{2}$ scaffold has equiaxed grains of size $10-20 \mathrm{~nm}$, which is governed by the size of the $\mathrm{TiO}_{2}$ mesopores, and they have relatively low crystallinity. In contrast, the grain size of $\mathrm{MAPbI}_{3}$ perovskite in the capping and the planar layers can be larger than $100 \mathrm{~nm}$, and the grains can be elongated and textured, with relatively high crystallinity; however, this grain size may depend on the specific thicknesses of the capping and the planar layers in other PSCs. $\mathrm{MAPbI}_{3}$ perovskite crystals within the mesoscopic region appear to be under hydrostatic tensile strain $(\sim 1 \%)$, which could be the result of their constrained crystallization within the mesoporous $\mathrm{TiO}_{2}$ scaffold. The dramatic differences in the crystal morphology and the size of the $\mathrm{MAPbI}_{3}$ perovskites in planar and mesoscopic-planar hybrid PSCs contribute in part to the important differences in their PSCs performance characteristics. This study highlights the use of TEM and XRD in revealing the crystal morphology of $\mathrm{MAPbI}_{3}$ perovskites in PSCs, which is critical to future efforts aimed at understanding effects of OTP morphology on the PSCs behavior and OTP morphological tailoring for improved PSCs performance.

\section{ASSOCIATED CONTENT}

\section{Supporting Information}

All experimental procedures involving synthesis, characterization, device fabrication, and testing. This material is available free of charge via the Internet The Supporting Information is available free of charge on the ACS Publications website at DOI: 10.1021 /acs.jpclett.5b00981.

\section{AUTHOR INFORMATION}

\section{Corresponding Authors}

*E-mail: yuanyuan_zhou@brown.edu (Y.Z.).

*E-mail: nitin_padture@brown.edu (N.P.P.).

\section{Notes}

The authors declare no competing financial interest.

"A.L.V. on leave from National Research Centre "Kruchatov Institute," Moscow 123182, Russia.

\section{ACKNOWLEDGMENTS}

Funding for the work at Brown University from the National Science Foundation (grant no. DMR-1305913) is gratefully acknowledged. S.P. acknowledges the research funding from the Chinese National Natural Science Foundation (grant no. 51202266). M.Y. and K.Z. acknowledge the support from the U.S. Department of Energy SunShot Initiative under the Next Generation Photovoltaics 3 program (DE-FOA-0000990) for the work performed at the National Renewable Energy Laboratory (contract no. DE-AC36-08-GO28308).

\section{REFERENCES}

(1) Snaith, H. J. Perovskites: The Emergence of a New Era for LowCost, High-Efficiency Solar Cells. J. Phys. Chem. Lett. 2013, 4, 36233630.

(2) Grätzel, M. The Light and Shade of Perovskite Solar Cells. Nat. Mater. 2014, 13, 838-842.

(3) Green, M. A.; Ho-Baillie, A.; Snaith, H. J. The Emergance of Perovskite Solar Cells. Nat. Photonics 2014, 8, 506-513.

(4) Jung, H. S.; Park, N.-G. Perovskite Solar Cells: From Materials to Devices. Small 2015, 11, 10-25.

(5) Kojima, A.; Teshima, K.; Shirai, Y.; Miyasaka, T. Organometal Halid Perovskites as Visible-Light Sensitizers for Photovoltaic Cells. J. Am. Chem. Soc. 2009, 131, 6050-6051.

(6) NREL. www.nrel.gov/ncpv/images/efficiency_chart.jpg, 2014.

(7) Zhao, Y.; Zhu, K. Solution-Chemistry Engineering toward HighEfficiency Perovskite Solar Cells. J. Phys. Chem. Lett. 2014, 5, 41754186.

(8) Kim, H.-S.; Lee, C.-R.; Im, J.-H.; Lee, K.-B.; Moehl, T.; Marchioro, A.; Moon, S.-J.; Humphrey-Baker, R.; Yum, J.-H.; Moser, J. E.; et al. Lead Iodide Perovskite Sensitized All-Solid-State Submicron Thin Film Mesoscopic Solar Cell with Efficiency Exceeding 9\%. Sci. Rep. 2012, 2, 591.

(9) Snaith, H. J.; Abate, A.; Ball, J. M.; Eperon, G. F.; Leijtens, T.; Noel, N. K.; Stranks, S. D.; Wang, J. T.-W.; Wojciechowski, K.; Zhang, W. Anomalous Hysteresis in Perovskite Solar Cells. J. Phys. Chem. Lett. 2014, 5, 1511-1515.

(10) Heo, J. H.; Im, S. H.; Noh, J. H.; Mandal, T. N.; Lim, C.-S.; Chang, J. A.; Lee, Y. H.; Kim, H.-J.; Sarkar, A.; Nazeeruddin, M. K.; et al. Efficient Inorganic-Organic Hybrid Heterojunction Solar Cells Containing Perokskite Compound and Polymeric Hole Conductors. Nat. Photonics 2013, 7, 486-491.

(11) Jeon, N. J.; Noh, J. H.; Yang, W. S.; Kim, Y. C.; Ryu, S.; Seo, J.; Seok, S. I. Compositional Engineering of Perovskite Materials for High-Performance Solar Cells. Nature 2015, 517, 476-480.

(12) Yang, W. S.; Noh, J. H.; Jeon, N. J.; Kim, Y. C.; Ryu, S.; Deo, J.; Seok, S. I. High-Performance Photovoltaic Perovskite Layers 
Fabricated through Intramolecular Exchange. Science 2015, 10.1126/ science.aaa9272.

(13) Eperon, G. E.; Burlakov, V. M.; Docampo, P.; Goriely, A.; Snaith, H. J. Morphological Control for High Performance, SolutionProcessed Planar Heterojunction Perovskite Solar Cells. Adv. Funct. Mater. 2014, 24, 151-157.

(14) Liang, P.-W.; Liao, V.-Y.; Chueh, C.-C.; Zuo, F.; Williams, S. T.; Xin, X.-K.; Lin, J.; Jen, A. K.-Y. Additive Enhanced Crystallization of Solution-Processed Perovskite for Highly Efficient Planar-Heterojunction Solar Cells. Adv. Mater. 2014, 26, 3748-3754.

(15) Grancini, G.; Marras, S.; Prato, M.; Giannini, C.; Quarti, C.; Angelis, F. D.; Bastiani, M. D.; Eperon, G. E.; Snaith, H. J.; Manna, L.; et al. The Impact of the Crystallization Process on the Structural and Optical Properties of Hybrid Perovskite Films for Photovoltaics. J. Phys. Chem. Lett. 2014, 5, 3836-3842.

(16) Listorti, A.; Juarez-Perez, E. J.; Frontera, C.; Roiati, V.; GarciaAndrade, L.; Colella, S.; Rizzo, A.; Ortiz, P.; Mora-Sero, I. Effect of Mesostructured Layer Upon Crystalline Properties and Device Performance on Perovskite Solar Cells. J. Phys. Chem. Lett. 2015, 6, $1628-1637$.

(17) Nanova, D.; Kast, A. K.; Pfannmoller, M.; Muller, C.; Veith, L.; Wacker, I.; Agari, M.; Hermes, W.; Erk, P.; Kowalsky, W.; Schroder, R. R.; Lovrincic, R. Unraveling the Nanoscale Morphologies of Mesoporous Perovskite Solar Cells and Their Correlation to Device Performance. Nano Lett. 2014, 14, 2735-2740.

(18) Zhou, Y.; Yang, M.; Vasiliev, A. L.; Garces, H. F.; Zhao, Y.; Wang, D.; Pang, S.; Zhu, K.; Padture, N. P. Growth Control of Compact $\mathrm{CH}_{3} \mathrm{NH}_{3} \mathrm{PbI}_{3}$ Thin Films Via Enhanced Solid-State Precursor Reaction for Efficient Planar Perovskite Solar Cells. J. Mater. Chem. A 2015, 3, 9249-9256.

(19) Xiao, M.; Huang, F.; Huang, W.; Dkhissi, Y.; Zhu, Y.; Etheridge, J.; Gray-Weale, A.; Bach, U.; Cheng, Y.-B.; Spiccia, L. A Fast Deposition-Crystallization Procedure for Highly Efficient Lead Iodiade Perovskite Thin-Film Solar Cells. Angew. Chem., Int. Ed. 2014, 53, 9898-9903.

(20) Zhou, Y.; Yang, M.; Wu, W.; Vasiliev, A. L.; Zhu, K.; Padture, N. P. Room-Temperature Crystallization of Hybrid-Perovskite Thin Films Via Solvent-Solvent Extraction for High-Performance Solar Cells. J. Mater. Chem. A 2015, 3, 8178-8184.

(21) Cullity, B. D. Elements of X-Ray Diffraction, 2nd ed.; AddisonWesley: Boston, MA, 1978.

(22) Scherer, G. W. Crystallization in Pores. Cem. Concr. Res. 1999, 29, 1347-1358.

(23) Jeon, N. J.; Noh, J. H.; Kim, Y. C.; Yang, W. S.; Ryu, S.; Seok, S. I. Solvent Engineering for High-Performance Inorganic-Organic Hybrid Perovskite Solar Cells. Nat. Mater. 2014, 9, 897-903.

(24) Kim, H.-S.; Park, N.-G. Parameters Affecting I-V Hysteresis of $\mathrm{CH}_{3} \mathrm{NH}_{3} \mathrm{PbI}_{3}$ Perovskite Solar Cells: Effect of Perovskite Crystal Size and Mesoporous $\mathrm{TiO}_{2}$ Layer. J. Phys. Chem. Lett. 2014, 5, 2927-2934.

(25) Frost, J. M.; Butler, K. T.; Brivio, F.; Hendon, C. H.; Schilfgaarde, M. v. Atomistic Origins of High-Performance in Hybrid Halide Perovskite Solar Cells. Nano Lett. 2014, 14, 2584-2591.

(26) Kutes, Y.; Ye, L.; Zhou, Y.; Pang, S.; Huey, B. D.; Padture, N. P. Direct Observation of Ferroelectrtic Domains in Solution-Processed $\mathrm{CH}_{3} \mathrm{NH}_{3} \mathrm{PbI}_{3}$ Perovskite Thin Films. J. Phys. Chem. Lett. 2014, 5, 3335-3339.

(27) Chen, H.-W.; Sakai, N.; Ikegami, M.; Miyasaka, T. Emergence of Hysteresis and Transient Ferroelectric Response in Organo-Lead Halide Perovskite Solar Cells. J. Phys. Chem. Lett. 2015, 6, 164-169.

(28) Lv, S.; Pang, S.; Zhou, Y.; Padture, N. P.; Hu, H.; Wang, L.; Zhou, X.; Zhu, H.; Zhang, L.; Huang, C.; Cui, G. One-Step, SolutionProcessed Formamidinium Lead Trihalide $(\mathrm{FAPbI}(3-\mathrm{x}) \mathrm{Clx})$ for Mesoscopic Perovskite-Polymer Solar Cells. Phys. Chem. Chem. Phys. 2014, 16, 19206-19211. 